\title{
BMJ Open Analysis of risk factors and outcome in peritoneal dialysis patients with early- onset peritonitis: a multicentre, retrospective cohort study
}

\author{
Xiaoyan Ma, ${ }^{1}$ Yingfeng Shi, ${ }^{1}$ Min Tao, ${ }^{1}$ Xiaolu Jiang, ${ }^{1}$ Yi Wang, ${ }^{1}$ Xiujuan Zang, ${ }^{2}$ \\ Lu Fang, ${ }^{1}$ Wei Jiang, ${ }^{1}$ Lin Du, ${ }^{1}$ Dewei Jin, ${ }^{1}$ Shougang Zhuang, ${ }^{1,3} \mathrm{Na} \mathrm{Liu}{ }^{1}$
}

To cite: Ma X, Shi Y, Tao M, et al. Analysis of risk factors and outcome in peritoneal dialysis patients with early-onset peritonitis: a multicentre, retrospective cohort study. BMJ Open 2020;10:e029949. doi:10.1136/ bmjopen-2019-029949

\section{- Prepublication history for} this paper is available online. To view these files, please visit the journal online (http://dx.doi. org/10.1136/bmjopen-2019029949).

Received 20 February 2019 Revised 13 October 2019 Accepted 14 January 2020

A Check for updates

C Author(s) (or their employer(s)) 2020. Re-use permitted under CC BY-NC. No commercial re-use. See rights and permissions. Published by BMJ.

${ }^{1}$ Department of Nephrology, Shanghai East Hospital, Tongji University School of Medicine, Shanghai, China

${ }^{2}$ Department of Nephrology, Shanghai Songjiang District Central Hospital, Shanghai, China

${ }^{3}$ Department of Medicine, Rhode Island Hospital, Providence, Rhode Island, USA

Correspondence to

Professor Na Liu;

naliubrown@163.com

\section{ABSTRACT}

Objectives To investigate the risk factors associated with early-onset peritonitis (EOP) and their influence on patients' technique survival and mortality.

Study design Retrospective, cohort study.

Setting Three peritoneal dialysis (PD) units in Shanghai. Participants PD patients from 1 June 2006 to 1 May 2018 were recruited and followed up until 31 December 2018. According to time-to-first episode of peritonitis, patients were divided into non-peritonitis $(n=144)$, EOP ( $\leq 6$ months, $n=74$ ) and late-onset peritonitis (LOP) ( $>6$ months, $n=139$ ).

Primary and secondary outcome measures EOP was defined as the first episode of peritonitis occurring within 6 months after the initiation of PD. The outcomes were allcause mortality and technique failure.

Results Of the 357 patients, 74 (20.7\%) patients developed their first episode of peritonitis within the first 6 months. Compared with the LOP group, the EOP group had older ages, more female patients, higher Charlson Comorbidity Index (CCl) score, lower serum albumin levels and renal function at the time of initiation of $P D$, and higher diabetes mellitus and peritonitis rates $(p<0.05)$. Staphylococcus was the most common Gram-positive organism in both EOP and LOP groups. The multivariate logistic regression analysis showed that factors associated with EOP included a higher CCI score (OR 1.285, $p=0.011$ ), lower serum albumin level (OR 0.924, $p=0.016)$ and lower Kt/N (OR 0.600, $p=0.018$ ) at start of PD. In the Cox proportional-hazards model, EOP was more likely a predictor of technique failure (HR 1.801, $\mathrm{p}=0.051)$. There was no difference between EOP and LOP for all-cause mortality.

Conclusion A higher $\mathrm{CCl}$ score and lower serum albumin level and Kt/V at PD initiation were significantly associated with EOP. EOP also predicted a high peritonitis rate and poor clinical outcome.

\section{INTRODUCTION}

In developing countries, the number of peritoneal dialysis (PD) patients has been increasing over time. ${ }^{12}$ PD-related peritonitis is a serious complication during PD therapy and remains the major reason for technique failure. ${ }^{3}$ Severe and prolonged peritonitis
Strengths and limitations of this study

- There was a strict exclusion criteria based on peritoneal dialysis histories.

- We conducted a multicentre study which ensured sufficient power in obtaining the risk factors of early-onset peritonitis.

- This was a retrospective cohort study, lacking of some objective information such as education level, economic development and living standard, which may cause bias.

- Our study lacked the adjustment of different centre factors (education, re-training and home visit) in the multivariate analysis.

- Although this was a multicentre study, the sample size was relatively small.

leads to structural and functional alterations of the peritoneal membrane, eventually leading to peritoneal fibrosis. ${ }^{4}$ Therefore, identification of the risk factors for peritonitis in the early stage of PD would help to reduce technique failures and mortality of PD.

The definition of early-onset peritonitis (EOP) varies widely between studies, which generally refers to PD-related peritonitis occurring within 3-24 months after surgical catheterisation. $^{5-8}$ Previous studies showed that the first episode of peritonitis in PD patients could significantly affect the prognosis of patients with end-stage renal disease (ESRD). ${ }^{9}$ However, few studies have specifically examined the risk factors for peritonitis in the early period of PD. And most of these were observational cohort studies carried out in single centre, ${ }^{5}{ }^{10} 11$ limiting the generalisability of their observed outcomes. To determine the risk factors for EOP in Chinese patients with chronic kidney disease and their influence on patients' technique survival and mortality, we conducted this multiple-centre, retrospective cohort study. 


\section{METHODS}

\section{Study population}

This was a multicentre retrospective cohort study that included 357 patients with ESRD who underwent PD in the Department of Nephrology in Baoshan Branch of Shanghai First People's Hospital, Shanghai Songjiang District Central Hospital and Shanghai East Hospital, Tongji University School of Medicine. All incident PD patients from 1 June 2006 to 1 May 2018 were recruited and followed up until 31 December 2018. The exclusion criteria were as follows: patients who had been using PD for fewer than 90 days, patients with an age younger than 18 years, and patients who initiated PD in other PD centres and previously accepted hemodialysis (HD) or kidney transplantation. There were 19 PD patients who suffered from peritonitis within the first 3 months: 6 subjects died, 3 patients were transferred to $\mathrm{HD}, 0$ patients underwent renal transplantation and 10 patients continued PD (these $10 \mathrm{PD}$ patients lacked information on peritoneal equilibration test). Patients were followed until any of the following events: death, a change to HD, renal transplantation or until 31 December 2018. According to the Chinese Peritoneal Dialysis Guideline, we adopted a standardised surgical catheterisation technique. ${ }^{12}$ We chose Tenckhoff silicone tube with double polyester sleeve. Double-purse string suture or double-layer suture was adopted to fix the catheter. Fine needle and thick line were used to prevent peripheral tube leakage. The exit direction of catheter tunnel was downward and outward, and the outer polyester sleeve was 2 to $3 \mathrm{~cm}$ away from the exit. All the surgical operations were performed in the operating room. The single-dose intravenous antibiotic $30 \mathrm{~min}$ before surgery was recommended to prevent infection. ${ }^{13}$ The first-generation or second-generation cephalosporin was suggested. ${ }^{13} 14$ According to the International Society for Peritoneal Dialysis (ISPD) peritonitis recommendations, ${ }^{13-15}$ we daily and topically applied mupirocin ointment to the catheter exit site to prevent exit-site infection. Patients initiated PD by Dianeal with $1.5 \%$ or $2.5 \%$ dextrose (Baxter Healthcare, Guangzhou, China). Dialysate concentration was $1.5 \%$ dextrose and replaced every 4 hours during the day, and $2.5 \%$ at night and kept in the body. A total of 213 patients had at least one episode of peritonitis. According to time-to-first episode of peritonitis, patients were divided into non-peritonitis $(\mathrm{n}=144)$, EOP ( $\leq 6$ months, $\mathrm{n}=74)$ and late-onset peritonitis (LOP) (>6 months, $\mathrm{n}=139$ ). We collected baseline characteristics within 1-3 months from the start of PD, including demographic data (age, gender, smoking, drinking, Charlson Comorbidity Index (CCI), body mass index (BMI)), medical history, drug-taking history, biochemical data (haemoglobin, serum electrolyte, fasting blood glucose, total cholesterol, total triglyceride, high-density lipoprotein cholesterol, low-density lipoprotein cholesterol, serum albumin, uric acid, creatinine, blood urea nitrogen, estimated glomerular filtration rate (eGFR), the clearance rate of urea nitrogen $(\mathrm{Kt} / \mathrm{V}))$, causes of ESRD and peritonitis episodes. Peritoneal fluid effluent from patients with peritonitis was collected and cultured for 1 to 5 days to identify the bacterial flora in the dialysate.

\section{Primary and secondary outcome measures}

EOP was defined as the first episode of peritonitis occurring within 6 months after the initiation of PD. This definition is consistent with other published articles. ${ }^{816}$ The outcomes were all-cause mortality and technique failure.

\section{Study definitions}

Diagnostic criteria for peritonitis are based on the 2010 ISPD guidelines. ${ }^{15}$ Patients diagnosed as peritonitis should meet at least two of the following three standards: (1) clinical symptoms or signs of peritonitis; (2) leucocyte count (at least $100 / \mathrm{mm}^{3}$ ) and polymorphonuclear neutrophilic cells proportion (at least $50 \%$ ) in peritoneal fluid effluent; (3) related pathogens in smear or culture of peritoneal fluid. EOP was defined as the first episode of peritonitis occurring within 6 months after the initiation of PD. The outcomes were all-cause mortality and technique failure. Death was an end-point event in the patient survival analysis. Relapse was defined as an episode occurring within 4 weeks of completion of therapy of a prior episode with the same organism ${ }^{13}$; recurrence referred to an episode occurring within 4 weeks of completion of therapy of a prior episode but with a different organism. ${ }^{13}$ Instead of transfer to HD therapy permanently, patients with both relapse and recurrence were treated by antibiotics and continued PD treatment. Complete cure was defined as the resolution of peritonitis without relapse or recurrence by antibiotics alone. ${ }^{7}$ However, some of the refractory peritonitis failed to clear up effluent after 5 days of appropriate antibiotics. This population of patients was transferred to HD permanently. We classified this population of patients into 'transfer to hemodialysis'. Other populations of patients who were transferred to HD were due to serious tunnel infection with peritonitis and ultrafiltration failure induced by encapsulating peritoneal sclerosis. Patients who transferred to HD were censored from the patient survival analysis, and death was censored for technique failure. Technique failure was defined as the transfer to HD therapy permanently (lasting for 30 days or more) due to ultrafiltration failure, peritonitis, exit-site infection and other operational problems. ${ }^{17}$

\section{Patient and public involvement}

No patient was involved in the design or conduct of the study, but the results of the study will be shared to patients coming for follow-up.

\section{Statistical analysis}

All statistical analyses were performed by using SPSS V.20.0 for Windows. The normal distributed data was shown as mean $\pm \mathrm{SD}$ and the skewed data was shown as median values with the 25th to 75 th percentile intervals. Categorical data was expressed as frequency (n) and percentage (\%). As for normally distributed data, Student's t-test was used for analysing the differences between the EOP group 
and LOP group, and one-way ANOVA for differences among non-peritonitis, EOP and LOP groups. Wilcoxon rank-sum test was used for skewed continuous data and the $\chi^{2}$ test or Fisher's exact test for categorical data. The Kaplan-Meier survival curves were drawn for each event of interest (technique survival and patient survival) and the log-rank test was used to compare curves. Univariate Cox proportional-hazards regression was used to select significant factors associated with study outcomes. Variables with $\mathrm{p}$ value $<0.10$ were selected for inclusion in the final multivariate Cox model. Multivariate logistic regression was calculated to select significant risk factors for EOP and the inclusion standard was also $\mathrm{p}$ value $<0.10$. Collinearity of variables was tested. A two-tailed $p$ value $<0.05$ was considered statistically significant.

\section{RESULTS}

\section{Patient characteristics}

A total of 357 patients with ESRD underwent continuous ambulatory peritoneal dialysis in three dialysis centres in Shanghai during the study period. All patients used Dianeal with $1.5 \%$ or $2.5 \%$ dextrose. The first episode of peritonitis was experienced by $74(20.7 \%)$ patients within 6 months after the start of PD: $11(11 / 61)$ in Shanghai East Hospital, 22 (22/142) in Shanghai Songjiang District Central Hospital and $41(41 / 154)$ in Baoshan Branch of Shanghai First People's Hospital. Median follow-up time for the 357 patients was 33.0 months (IQR 14.0-50.0 months). There were 211 men $(59.1 \%)$ with an average age of $61.6 \pm 14.0$ years and 145 women $(40.9 \%)$ with an average age of $65.3 \pm 12.9$ years. The most common primary renal diseases were chronic glomerulonephritis $(43.1 \%)$ and diabetic nephropathy (34.2\%). Compared with the patients with LOP, the EOP patient group had older ages, more female patients, higher CCI score and lower serum albumin levels, renal function and Kt/V at PD initiation and higher diabetes mellitus $(p<0.05)$. The percentage of patients who experienced more than three peritonitis episodes in EOP group $(55.4 \%)$ was higher than LOP group (33.8\%). Additional demographic and laboratory characteristics of the study population were presented in table 1 .

\section{Causative organisms}

In table 2, among 213 patients with peritonitis, 47 (22.1\%) were due to Gram-positive organisms, 24 (11.3\%) were due to Gram-negative organisms, $6(2.8 \%)$ were due to fungi, $1(0.4 \%)$ was due to multiple organisms and 135 (63.4\%) were culture-negative. Staphylococcus was the most common Gram-positive organism in both groups. Compared with the EOP patient group, the LOP patient group had more culture-negative peritonitis $(89.2 \%$ vs $14.9 \%, \mathrm{p}<0.001)$. The incidences of culture-negative peritonitis were $37.1 \%(13 / 35)$ in Shanghai East Hospital, $71.7 \%(38 / 53)$ in Shanghai Songjiang District Central Hospital and $67.2 \%(84 / 125)$ in Baoshan Branch of Shanghai First People's Hospital $(\mathrm{p}=0.002)$.

\section{Outcomes}

The total peritonitis rate (in a population that included EOP group, LOP group and peritonitis-free group) was 0.490 episodes per patient-year (213 patients presented 509 episodes of peritonitis during 1039.58 patient-years of follow-up). The peritonitis rate (in a population that included EOP group and LOP group) was 0.660 episodes per patient-year (213 patients presented 509 episodes of peritonitis during 771.33 patient-years of follow-up). The peritonitis rate in EOP group was 0.960 episodes per patient-year (74 patients presented 209 episodes of peritonitis during 217.75 patient-years of follow-up). The peritonitis rate in LOP group was 0.542 episodes per patient-year (139 patients presented 300 episodes of peritonitis during 553.58 patient-years of follow-up). The peritonitis rates in Shanghai East Hospital, Shanghai Songjiang District Central Hospital and Baoshan Branch of Shanghai First People's Hospital were $0.41,0.31$ and 0.61 episodes per patient-year, respectively. Early-onset first episode of peritonitis had a lower cure rate $(17.6 \%$ vs $33.8 \%$, table 2$)$, higher rate of transferring to $\mathrm{HD}(27.0 \%$ vs $19.4 \%$, table 2$)$ and higher mortality $(21.6 \%$ vs $14.4 \%$, table 2$)$ compared with late-onset first episode of peritonitis.

\section{Technique failure}

The variables including time to first peritonitis (EOP vs LOP), age, sex, smoking, drinking, CCI, BMI, haemoglobin, total cholesterol, total triglyceride, serum albumin, total $\mathrm{Kt} / \mathrm{V}$ and diabetes were calculated into the Cox proportional-hazards model for technique failure. We found that EOP was associated with technique failure compared with the LOP group, with a HR of 1.801 (table 3, p=0.051). Kaplan-Meier analysis showed that compared with LOP group, technique survival was lower in the EOP group (log rank 3.943, $\mathrm{p}=0.047$, figure 1).

\section{All-cause mortality}

During the study period, a total of 52 patients died: 16 patients in the EOP group and 20 patients in the LOP group. Variables with $\mathrm{p}$ value $<0.10$ in univariate Cox regression analysis, including the time to first peritonitis (EOP vs LOP), age, serum albumin and total Kt/V, were chosen for further adjustment in multivariate Cox proportional-hazards model. After adjustment, there was no significant difference between the EOP and LOP groups (table 3). Figure 2 described cumulative survival by EOP and LOP groups using the Kaplan-Meier analysis. Compared with LOP group, cumulative survival was lower in the EOP group $(\log$ rank 4.060, $\mathrm{p}=0.044)$.

\section{Risk factors of EOP}

Variables in table 1 were tried in a univariate logistic regression model, and only variables with $\mathrm{p}$ value $<0.10$ for peritonitis were depicted in table 4 . Based on the simple logistic regression analysis of risk factors associated with EOP, we constructed a multiple logistic regression model using variables including gender, age, CCI score, diabetes, serum albumin and Kt/V. We found that higher 
Table 1 Baseline characteristic of the study population

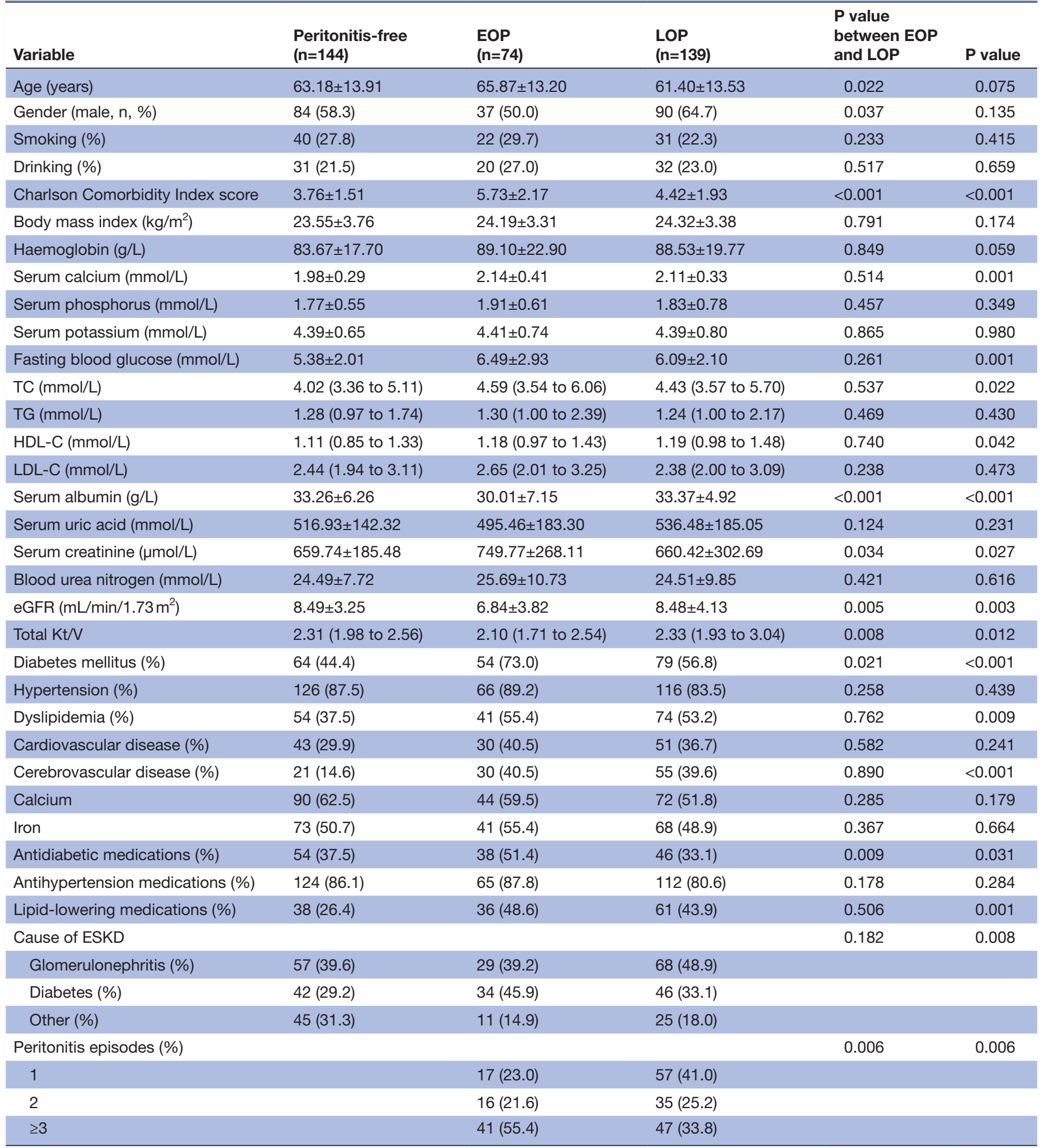

eGFR, estimated glomerular filtration rate; EOP, early-onset peritonitis; ESKD, end-stage kidney disease; HDL-C, high-density lipoprotein cholesterol; LDL-C, low-density lipoprotein cholesterol; LOP, late-onset peritonitis; TC, total cholesterol; TG, total triglyceride.

CCI score (OR 1.285, 95\% CI 1.058 to $1.561, \mathrm{p}=0.011$ ), lower serum albumin level (OR $0.924,95 \%$ CI 0.867 to $0.985, \mathrm{p}=0.016$ ) and $\mathrm{Kt} / \mathrm{V}$ (OR $0.600,95 \%$ CI 0.394 to $0.915, \mathrm{p}=0.018$ ) at the start of PD were significantly associated with EOP (table 4 ).

\section{DISCUSSION}

Our retrospective cohort study of $357 \mathrm{PD}$ patients showed that $74(20.7 \%)$ patients in three Shanghai dialysis centres developed the first episodes of peritonitis within the first 6 months. Higher CCI score, lower serum albumin level 
Table 2 Organism and outcome of different vintages of peritonitis (n, \%)

\begin{tabular}{|cccc}
\hline Causative organisms & $\begin{array}{l}\text { Early-onset peritonitis } \\
\text { episodes } \mathbf{( n )}\end{array}$ & $\begin{array}{l}\text { Late-onset peritonitis } \\
\text { episodes } \mathbf{n} \text { ) }\end{array}$ & P value \\
\hline $\begin{array}{l}\text { Causative organisms } \\
\text { Gram-positive organisms }\end{array}$ & $38(51.4)$ & $9(6.5)$ & $<0.001$ \\
\hline Staphylococcus aureus & $7(18.4)$ & $0(0.0)$ & 0.163 \\
\hline Coagulase-negative & $3(7.9)$ & $0(0.0)$ & 0.384 \\
\hline Staphylococcus & $16(42.1)$ & $8(88.9)$ & 0.012 \\
\hline Streptococcus sp & $4(10.5)$ & $1(11.1)$ & 0.959 \\
\hline Enterococcus sp & $4(10.5)$ & $0(0.0)$ & 0.309 \\
\hline Other Gram-positives & $4(10.5)$ & $0(0.0)$ & 0.309 \\
\hline Gram-negative organisms & $20(27.0)$ & $4(2.9)$ & $<0.001$ \\
\hline Escherichia coli & $8(40.0)$ & $0(0.0)$ & 0.121 \\
\hline Klebsiella sp & $6(30.0)$ & $1(25.0)$ & 0.841 \\
\hline Acinetobacter sp & $4(20.0)$ & $1(25.0)$ & 0.822 \\
\hline Pseudomonas aeruginosa & $2(10.0)$ & $1(25.0)$ & 0.408 \\
\hline Other Gram-negatives & $0(0.0)$ & $1(25.0)$ & 0.022 \\
\hline Fungi & $4(5.4)$ & $2(1.4)$ & 0.096 \\
\hline Multiple organisms & $1(1.4)$ & $0(0.0)$ & 0.170 \\
\hline Culture-negative peritonitis & $11(14.9)$ & $124(89.2)$ & $<0.001$ \\
\hline Outcomes & & & 0.063 \\
\hline Complete cure & $13(17.6)$ & $47(33.8)$ & \\
\hline Relapse or recurrence & $25(33.8)$ & $27(32.4)$ & $20(14.4)$ \\
\hline Transfer to hemodialysis & $20(27.0)$ & & \\
\hline Death & $16(21.6)$ & & \\
\hline
\end{tabular}

and $\mathrm{Kt} / \mathrm{V}$ at the start of PD were significantly associated with EOP. In addition, an early peritonitis onset predicted a high peritonitis rate and technique failure.

EOP is a major complication of PD, directly or indirectly causing the abandon of dialysis treatment. In this study, among 213 patients with peritonitis, $47(22.1 \%)$ were due to Gram-positive organisms, $24(11.3 \%)$ were due to Gram-negative organisms and $6(2.8 \%)$ were due to fungi. Staphylococcus was the most common Grampositive organism in both EOP and LOP. This bacterial flora distribution and high incidence of Staphylococcus were similar to previous reports. ${ }^{5} 1819$ Fungal peritonitis was rare in PD patients, but could bring out irreversible peritoneal damage. ${ }^{20}$ Recent clinical studies confirmed that the incidence of fungal peritonitis was only $3 \%-6 \%,{ }^{20}$ while the relative mortality rate was up to $20 \%-30 \% .^{21}$ The culture-negative proportion for the first peritonitis episode was high in patients with LOP $(89.2 \%)$. Also, the incidences of culture-negative peritonitis were $37.1 \%$ $(13 / 35)$ in Shanghai East Hospital, $71.7 \%$ (38/53) in Shanghai Songjiang District Central Hospital and 67.2\% (84/125) in Baoshan Branch of Shanghai First People's Hospital $(p=0.002)$. The high culture-negative proportion may primarily attribute to early antibiotic treatment and limited effluent culture technique in small-scale PD units. Before 2014, the technology of blood culture for
PD effluent has not been widely adopted by small-scale district hospitals in Shanghai. In the district PD units, dialysate was inoculated onto solid medium and then incubated only in aerobic environment. It accounted for about $60 \%$ of patients with culture-negative peritonitis in this investigation. Since 2015, all these three units in Shanghai chose blood-culture bottle for the preferred technique to culture micro-organisms in PD effluent. Lacking centrifugation of PD effluent and recent antibiotic usage may be the major reasons for the rest of $40 \%$ negative effluent cultures in this investigation. In addition, culture-negative peritonitis was higher in LOP than EOP group in the same study period because patients with LOP underwent dialysis more than 6 months and had more experience in PD. In the early stage of peritonitis, some of the experienced patients with PD might take dialysate to wash the peritoneum to relieve abdominal pain. Diluted peritoneal fluid would result in a high negative rate of peritoneal effluent culture. Considering the high culture-negative rate in this study, our three PD units will take a series of measures to improve our culture methods, including centrifugation of PD effluent, incubation in aerobic, microaerophilic and anaerobic environments, using antibiotic neutralisation bottle and so on. ${ }^{13} 14$

By the end of the study, 509 episodes of peritonitis occurred in 213 patients, and the peritonitis rate was 
Table 3 Cox proportional-hazards model for technique failure and patient mortality

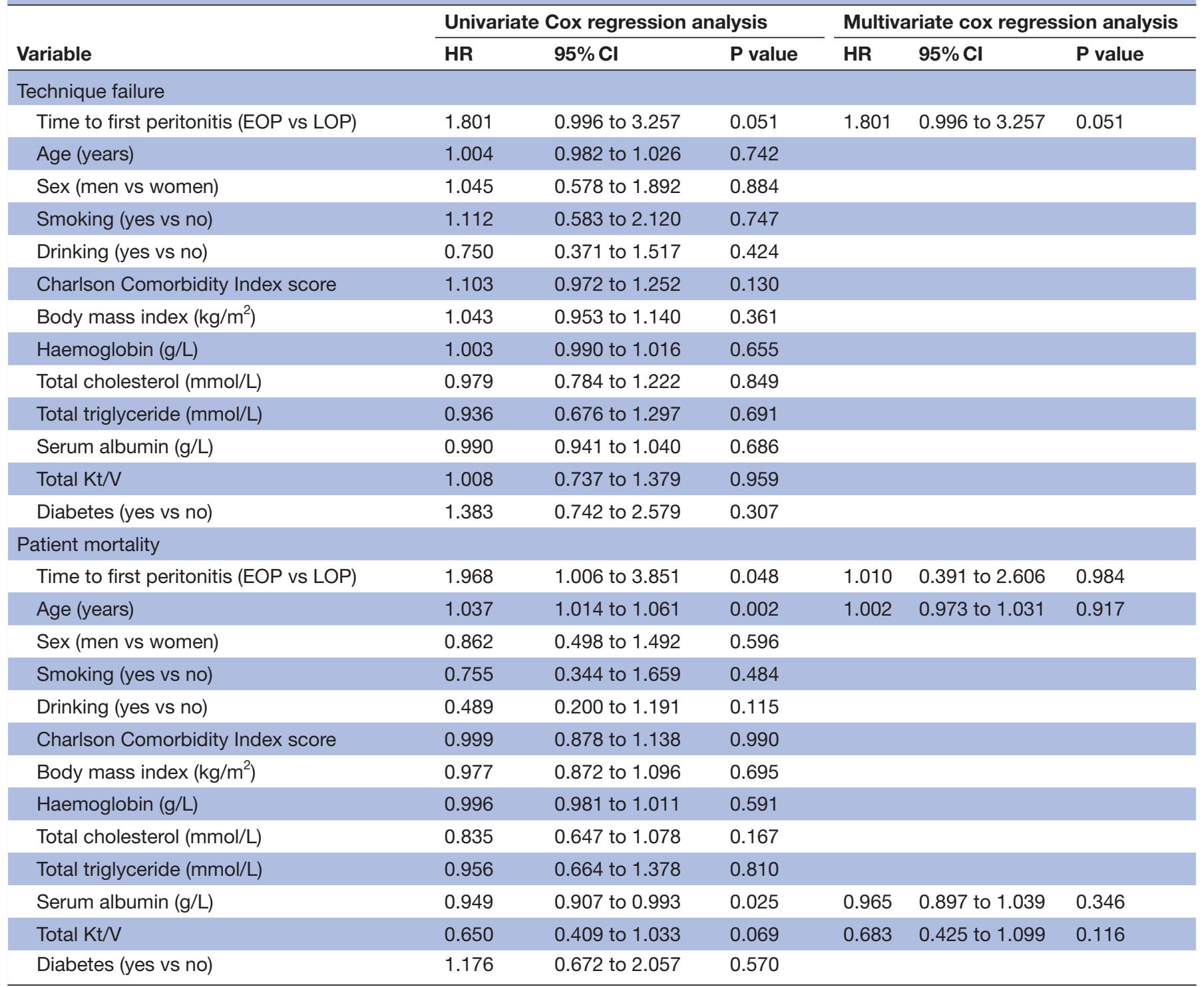

0.490 episodes per patient-year. The peritonitis rates in Shanghai East Hospital, Shanghai Songjiang District Central Hospital and Baoshan Branch of Shanghai First People's Hospital were $0.41,0.31$ and 0.61 episodes per patient-year, respectively. Recently, some investigations from other areas of China have indicated that the peritonitis rate was 0.196 episodes per patient-year in Taiwan, ${ }^{5}$ 0.158 episodes per patient-year in Guangzhou, ${ }^{7} 0.296$ episodes per patient-year in Suzhou ${ }^{16}$ and 0.158 per patient-year in Hangzhou. ${ }^{8}$ Peritonitis rate in our study was higher than the rest of China. Among the patients with EOP who had $\geq 3$ episodes of peritonitis, 25 patients from the EOP group experienced recurrent peritonitis and 16 patients from the EOP group experienced repeat peritonitis. Also, 43.8\% repeat patients had staphylococcal peritonitis; $75 \%$ of patients with EOP with $\geq 3$ episodes of peritonitis came from Baoshan Branch of Shanghai First People's Hospital. Most of them are fishermen and live in the Chongming Island. Because of the poorer economic abilities and living conditions, they can easily suffer from malnutrition and peritonitis again. ${ }^{22}{ }^{23}$ In addition, lack of home visit by PD nurses makes it difficult to determine which patients require $\mathrm{PD}$ re-training. Lacking of technical improvement in small-scale PD units is also an important reason for high peritonitis rate.

The complete cure rate in our study was related low (EOP $17.6 \%$, LOP $33.8 \%$ ). All the patients with PD from these three centres received prophylactic intravenous antibiotics prior to PD catheter insertion. However, most antibiotics used are first-generation or second-generation cephalosporin. They may not cover all the Gram-negative organisms, resulting in increased rate of relapse and recurrence. To address this issue, we may have to modify our empirical antibiotic regimen by using more effective antibiotics such as third-generation cephalosporin and applying individualised treatment strategy. In addition, patients with poorer economic abilities and living conditions can easily suffer from malnutrition and peritonitis 


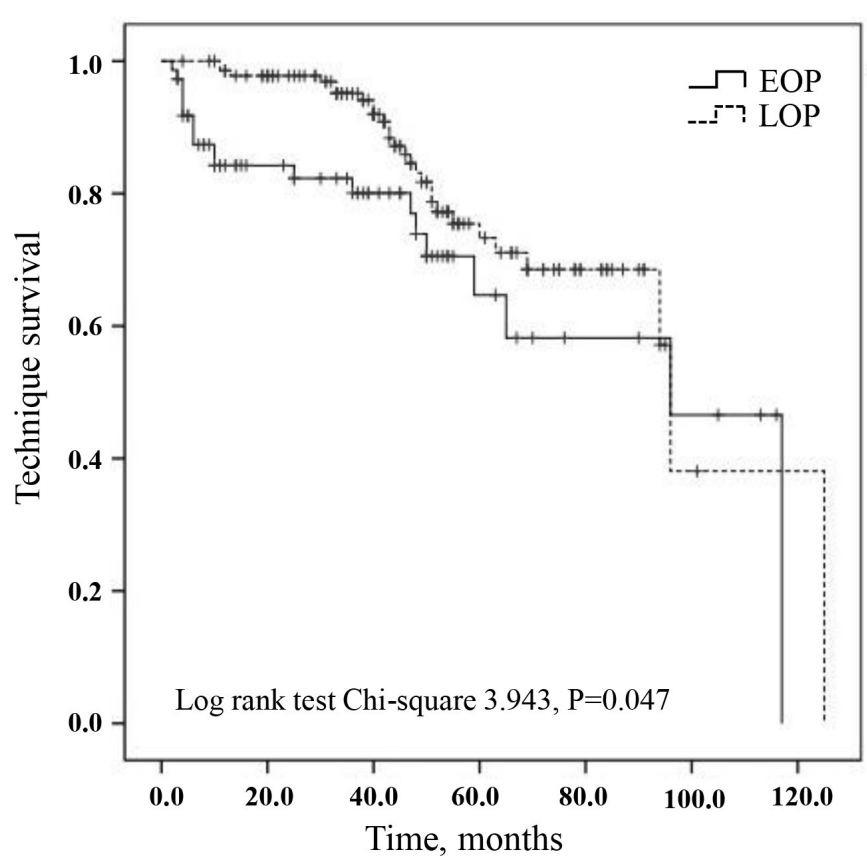

Figure 1 Technique survival according to early-onset peritonitis (EOP) and late-onset peritonitis (LOP). Death was censored form the technique survival analysis. Log-rank test $\chi^{2} 3.943, p=0.047$.

again. ${ }^{22} 23$ Finally, the reason for the low cure rate in this study may also include a considerable number of patients with HD due to other dialysis-related complications.

Our study indicated that lower serum albumin was one of the major risk factors for EOP. Loss of protein would cause negative nitrogen balance and malnutrition, leading to a decline in immune function and increased susceptibility

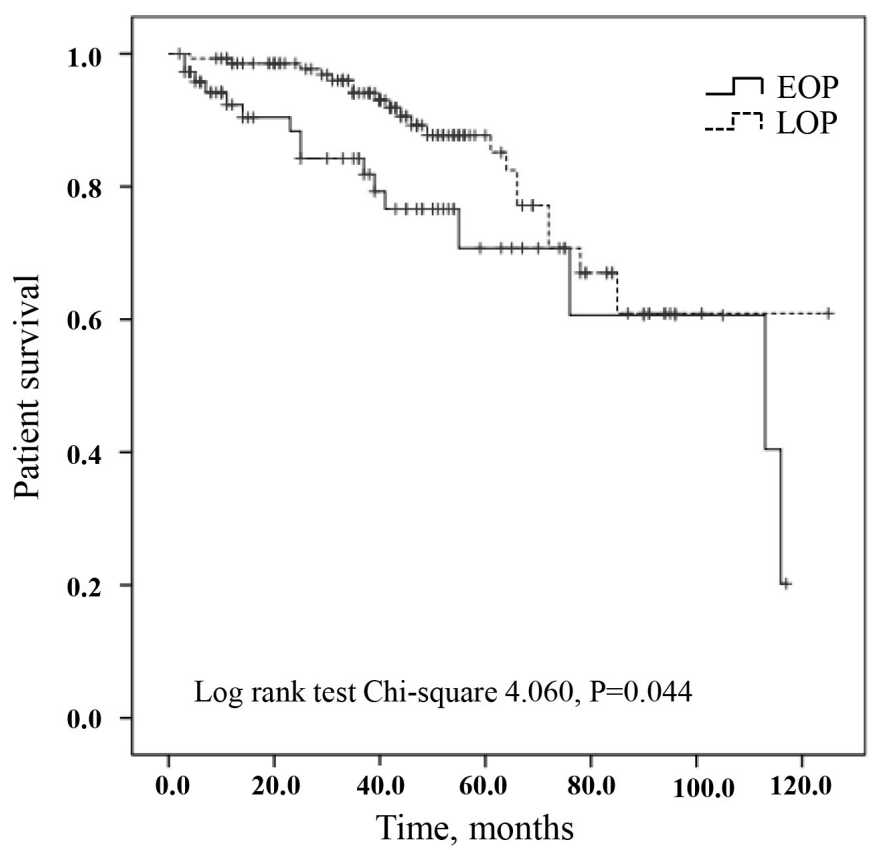

Figure 2 Patient survival according to early-onset peritonitis (EOP) and late-onset peritonitis (LOP). Patients who transferred to hemodialysis were censored form the patient survival analysis. Log-rank test $\chi^{2} 4.060, p=0.044$. to pathogenic micro-organisms. ${ }^{24}$ Malnutrition was one of the most common complications in patients with $\mathrm{PD}$, and plasma albumin level was an important clinical predictor. Hypoalbuminemia was proved to be related with malnutrition, protein losses and inflammation. ${ }^{25}{ }^{26}$ Wang Qin et al discovered that patients with an initial serum albumin level less than $2.9 \mathrm{~g} / \mathrm{dL}$ had a higher incidence of peritonitis and regarded hypoalbuminemia as an independent predictor for subsequent peritonitis at the start of PD therapy. ${ }^{23}$ Further studies demonstrated that low serum albumin level increased all-cause, cardiovascular and infection-related mortality in both PD and HD patients. ${ }^{27}$ In addition to peritoneal infection, hypoalbuminemia was also found to be associated with septicaemia, pneumonia and other inflammatory responses. ${ }^{28-32}$ In this study, we reaffirmed that a low baseline serum albumin level was an independent risk factors for EOP (OR 0.924, 95\% CI 0.867 to $0.985, \mathrm{p}=0.016$ ).

Although older age was not an independent risk factor for EOP, baseline data showed that patients in EOP group were older than those in the LOP group (65.87 \pm 13.20 vs $61.40 \pm 13.53, p=0.022)$. It was reported that elderly patients were more likely to progress to a worse outcome, including HD, renal transplantation or death. ${ }^{33}$ Incidence of malnutrition in elderly patients with PD was more common than young and middle-aged patients. Together with cardiovascular diseases, cerebrovascular diseases, hearing and visual impairments, all of these factors increase and aggravate the episode of peritonitis. $^{34-36}$ Malnutrition in the elderly affected the quality of dialysis patients' life and also was an important factor in comorbidity and mortality. ${ }^{37}$ Other elements that increased peritonitis susceptibility in elderly patients included generalised functional deterioration, weakened immune system, ${ }^{38}$ combined chronic diseases, bad eyesight, poor aseptic concept, lack of compliance and living alone. Their atypical clinical symptoms of peritonitis could be regarded as another essential reason. Upregulated pain threshold, unobtrusive bellyache and mild subjective symptoms might cover up EOP until the occurrence of liquid turbidity, which would delay the best time for treatment.

Comparison in biochemical indicators revealed that $\mathrm{Kt} / \mathrm{V}$ and residual renal function decreased significantly after EOP. Multivariate logistic regression showed that a lower total Kt/V (OR $0.600,95 \%$ CI 0.394 to 0.915 , $\mathrm{p}=0.018$ ) at the start of PD was associated with EOP. These results suggest that early infection with peritonitis might further worsen renal function, especially the scavenging capacity of solutes by residual kidney. Early inflammatory response and renal function damage might be the underlying causes of peritonitis. Some studies suggested that the survival rate of patients with PD depends more on residual renal function than the peritoneal cleaning capacity. ${ }^{39-41}$ Harris et al further put forward that residual renal function less than $4 \mathrm{~mL} /$ $\min / 1.73 \mathrm{~m}^{2}$ was associated with high mortality during PD. ${ }^{42}$ Therefore, we should pay close attention to the 
Table 4 Logistic regression analysis of factors associated with early-onset peritonitis

\begin{tabular}{|c|c|c|c|c|c|c|}
\hline \multirow[b]{2}{*}{ Variable } & \multicolumn{3}{|c|}{ Univariate logistic regression analysis } & \multicolumn{3}{|c|}{ Multivariate logistic regression analysis } \\
\hline & OR & $95 \% \mathrm{Cl}$ & $P$ value & OR & $95 \% \mathrm{Cl}$ & $P$ value \\
\hline Sex (men vs women) & 0.544 & 0.307 to 0.966 & 0.038 & 0.586 & 0.295 to 1.163 & 0.126 \\
\hline Charlson Comorbidity Index score & 1.355 & 1.173 to 1.566 & $<0.001$ & 1.285 & 1.058 to 1.561 & 0.011 \\
\hline Diabetes & 2.051 & 1.111 to 3.786 & 0.022 & 1.084 & 0.457 to 2.571 & 0.854 \\
\hline Total Kt/N & 0.553 & 0.370 to 0.827 & 0.004 & 0.600 & 0.394 to 0.915 & 0.018 \\
\hline
\end{tabular}

change of residual renal function when monitoring the adequacy of dialysis.

The relationship between peritonitis and technique failure and death has been investigated in previous Chinese single-centre studies. ${ }^{78}$ A study in Chinese Zhejiang province showed that EOP was a significant predictor of allcause mortality. As for technique failure, they found no significant difference between EOP and LOP. ${ }^{8}$ However, a study in Chinese Guangzhou province indicated that technique failure in EOP group was lower than LOP group, but patient survival did not differ between two groups. ${ }^{7}$ Our present study showed that EOP was more likely a predictor of technique failure (HR 1.801, 95\% CI 0.996 to $3.257, \mathrm{p}=0.051$ ). There was no difference between EOP and LOP for all-cause mortality. These conclusions might be limited by regional and demographic differences in different dialysis centres. However, all three studies indicated that patients who experienced peritonitis early after the initiation of PD tend to experience more episodes of peritonitis. Repeating peritonitis in patients with EOP damages membrane permeability and reduces ultrafiltration, and also increases severe systemic inflammation, leading to worse clinical outcomes. ${ }^{43}$ Thus, appropriately dealing with the risk factors of EOP will be good to reduce infection incidence, raise therapeutic effect of PD and improve patient's life quality and prognosis.

There are several limitations to this study. First, this was a retrospective cohort study, lacking some objective information such as education level, economic development and living standard, which may cause bias. Second, our study lacked the adjustment of different centre factors (education, re-training and home visit) in the multivariate analysis. Third, although this was a multicentre study, the sample size was relatively small. Further larger size and prospective investigation are necessary.

\section{CONCLUSION}

This retrospective cohort study found that a higher CCI score and lower serum albumin and $\mathrm{Kt} / \mathrm{V}$ at PD initiation were significantly associated with EOP. In addition, an early peritonitis onset predicted a high peritonitis rate and worse clinical outcomes. Understanding the risk factors for EOP will help to develop effective measures to prevent or delay the complication of peritoneal dialysis as much as possible.

Acknowledgements The authors appreciate all the participants and their families. They also thank the members of the study team from Shanghai East Hospital Affiliated to Tongji University School of Medicine, Shanghai Songjiang District Central Hospital and Baoshan Branch of Shanghai First People's Hospital for their assistance in completing this project.

Contributors XM, YS, MT and XJ contributed equally to this work. XM, YS, MT and $\mathrm{XJ}$ performed the statistical analysis and wrote the manuscript; XM, YS, MT, XJ, YW, DJ, LF, WJ, LD and XZ participated in the data collection; XM, YS, SZ and NL contributed to discussion; XM, SZ and NL participated in the design of the study and edited the manuscript. All authors contributed to data interpretation and revisions of the manuscript critically for important intellectual content. All authors approved the final version of the submitted manuscript and agreed to be accountable for all aspects of the work in ensuring that questions related to the accuracy or integrity of any part of work are appropriately investigated and resolved.

Funding This study was supported by the National Nature Science Foundation of China grants (81670690, 81470991 and 81200492 to NL, 81270778, 81470920, 81670623 and 81830021 to SZ), the Key Discipline Construction Project of Pudong Health Bureau of Shanghai (PWZxk2017-05 to NL), the Science Technology grant of Jiangxi Province Municipal Health Commission (20184077 to LF), the Branch grant of National key grants of Ministry of Science and Technology (2018YFA0108802 to SZ), the US National Institutes of Health (2R01DK08506505A1 to SZ) and the Shanghai Scientific Committee of China (13PJ1406900 to NL).

\section{Competing interests None declared.}

Patient consent for publication Not required.

Ethics approval The study was conducted according to the guidelines of the Helsinki Declaration and was approved by the Human Research Ethics Committee of Shanghai East Hospital Affiliated to Tongji University School of Medicine, Human Research Ethics Committee of Shanghai Songjiang District Central Hospital and the Human Research Ethics Committee of Baoshan Branch of Shanghai First People's Hospital. The human research ethics committees approved this study and agreed to collect the information from the hospital databases. They waived the need for participant consent.

Provenance and peer review Not commissioned; externally peer reviewed.

Data availability statement The data sets generated and analysed during the current study are available from the corresponding author on reasonable request.

Open access This is an open access article distributed in accordance with the Creative Commons Attribution Non Commercial (CC BY-NC 4.0) license, which permits others to distribute, remix, adapt, build upon this work non-commercially, and license their derivative works on different terms, provided the original work is properly cited, appropriate credit is given, any changes made indicated, and the use is non-commercial. See: http://creativecommons.org/licenses/by-nc/4.0/.

\section{REFERENCES}

1 Li PK-T, Chow KM, Van de Luijtgaarden MWM, et al. Changes in the worldwide epidemiology of peritoneal dialysis. Nat Rev Nephrol 2017;13:90-103. 
2 Jain AK, Blake P, Cordy P, et al. Global trends in rates of peritoneal dialysis. J Am Soc Nephrol 2012;23:533-44.

3 Cho Y, Johnson DW. Peritoneal dialysis-related peritonitis: towards improving evidence, practices, and outcomes. Am J Kidney Dis 2014;64:278-89.

4 Thirugnanasambathan T, Hawley CM, Badve SV, et al. Repeated peritoneal dialysis-associated peritonitis: a multicenter registry study. Am J Kidney Dis 2012;59:84-91.

5 Hsieh Y-P, Wang S-C, Chang C-C, et al. The negative impact of early peritonitis on continuous ambulatory peritoneal dialysis patients. Perit Dial Int 2014;34:627-35.

6 See EJ, Johnson DW, Hawley CM, et al. Early peritonitis and its outcome in incident peritoneal dialysis patients. Perit Dial Int 2017. doi:10.3747/pdi.2017.00029. [Epub ahead of print: 28 Sep 2017].

$7 \mathrm{Wu} \mathrm{H}$, Huang R, Yi C, et al. Risk factors for early-onset peritonitis in southern Chinese peritoneal dialysis patients. Perit Dial Int 2016;36:640-6.

8 Tian Y, Xie X, Xiang S, et al. Risk factors and outcomes of early-onset peritonitis in Chinese peritoneal dialysis patients. Kidney Blood Press Res 2017;42:1266-76.

9 Béchade C, Guittet L, Evans D, et al. Early failure in patients starting peritoneal dialysis: a competing risks approach. Nephrol Dial Transplant 2014:29:2127-35.

10 Feng S, Wang Y, Qu B, et al. Impact of early-onset peritonitis on mortality and technique survival in peritoneal dialysis patients. Springerplus 2016;5:1676.

11 Fourtounas C, Savidaki E, Dousdabanis P, et al. Peritonitis during the first year after commencement of peritoneal dialysis has an impact on technique survival and patient morbidity. Adv Perit Dial 2006;22:50-4.

12 Catheterization CEGoPD. Chinese guidelines for peritoneal dialysis catheterization. Chinese J Nephrol 2016;32:867-71.

13 PK L, Szeto CC, Piraino B, et al. ISPD peritonitis recommendations: 2016 update on prevention and treatment. Perit Dial Int 2016;36:481-508.

14 Szeto C-C, Li PK-T, Johnson DW, et al. ISPD catheter-related infection recommendations: 2017 update. Perit Dial Int 2017;37:141-54.

15 Li PK-T, Szeto CC, Piraino B, et al. Peritoneal dialysis-related infections recommendations: 2010 update. Perit Dial Int 2010;30:393-423.

16 Wang Z, Jiang L, Feng S, et al. Early peritonitis is an independent risk factor for mortality in elderly peritoneal dialysis patients. Kidney Blood Press Res 2015;40:298-305.

17 Shen JI, Mitani AA, Saxena AB, et al. Determinants of peritoneal dialysis technique failure in incident US patients. Perit Dial Int 2013;33:155-66.

18 Barretti P, Doles JVP, Pinotti DG, et al. Efficacy of antibiotic therapy for peritoneal dialysis-associated peritonitis: a proportional metaanalysis. BMC Infect Dis 2014;14:445.

19 Govindarajulu S, Hawley CM, McDonald SP, et al. Staphylococcus aureus peritonitis in Australian peritoneal dialysis patients: predictors, treatment, and outcomes in 503 cases. Perit Dial Int 2010;30:311-9.

20 Matuszkiewicz-Rowinska J. Update on fungal peritonitis and its treatment. Perit Dial Int 2009;29:S161-5.

21 Szeto C-C, Chow K-M. Gram-negative peritonitis - the Achilles heel of peritoneal dialysis? Perit Dial Int 2007;27:S267-71.

22 Prasad N, Gupta A, Sharma RK, et al. Impact of nutritional status on peritonitis in CAPD patients. Perit Dial Int 2007;27:42-7.

23 Wang Q, Bernardini J, Piraino B, et al. Albumin at the start of peritoneal dialysis predicts the development of peritonitis. Am J Kidney Dis 2003;41:664-9.
24 Li Z-J, An X, Mao H-P, et al. Association between depression and malnutrition-inflammation complex syndrome in patients with continuous ambulatory peritoneal dialysis. Int Urol Nephrol 2011;43:875-82.

25 Fanali G, di Masi A, Trezza V, et al. Human serum albumin: from bench to bedside. Mol Aspects Med 2012;33:209-90.

26 Yu Z, Tan BK, Dainty S, et al. Hypoalbuminaemia, systemic albumin leak and endothelial dysfunction in peritoneal dialysis patients. Nephrol Dial Transplant 2012;27:4437-45.

27 Mehrotra R, Duong U, Jiwakanon S, et al. Serum albumin as a predictor of mortality in peritoneal dialysis: comparisons with hemodialysis. Am J Kidney Dis 2011;58:418-28.

28 Seo MH, Choa M, You JS, et al. Hypoalbuminemia, low base excess values, and tachypnea predict 28 -day mortality in severe sepsis and septic shock patients in the emergency department. Yonsei Med $\mathrm{J}$ 2016;57:1361-9.

29 Mizuno T, Mizokami F, Fukami K, et al. The influence of severe hypoalbuminemia on the half-life of vancomycin in elderly patients with methicillin-resistant Staphylococcus aureus hospital-acquired pneumonia. Clin Interv Aging 2013;8:1323-8.

30 Juneja M, Baidoo L, Schwartz MB, et al. Geriatric inflammatory bowel disease: phenotypic presentation, treatment patterns, nutritional status, outcomes, and comorbidity. Dig Dis Sci 2012;57:2408-15.

31 Don BR, Kaysen G. Serum albumin: relationship to inflammation and nutrition. Semin Dial 2004;17:432-7.

32 Magnussen B, Oren Gradel K, Gorm Jensen T, et al. Association between hypoalbuminaemia and mortality in patients with community-acquired bacteraemia is primarily related to acute disorders. PLoS One 2016;11:e0160466.

33 Maitra S, Burkart J, Fine A, et al. Patients on chronic peritoneal dialysis for ten years or more in North America. Perit Dial Int 2000;20:S127.

34 Sakacı T, Ahbap E, Koc Y, et al. Clinical outcomes and mortality in elderly peritoneal dialysis patients. Clinics 2015;70:363-8.

35 Valderrábano F, Jofre R, López-Gómez JM. Quality of life in endstage renal disease patients. Am J Kidney Dis 2001;38:443-64.

36 Joly D, Anglicheau D, Alberti C, et al. Octogenarians reaching endstage renal disease: cohort study of decision-making and clinical outcomes. J Am Soc Nephrol 2003;14:1012-21.

37 Tennankore KK, Bargman JM. Nutrition and the kidney: recommendations for peritoneal dialysis. Adv Chronic Kidney Dis 2013;20:190-201.

38 Hsieh Y-P, Chang C-C, Wen Y-K, et al. Predictors of peritonitis and the impact of peritonitis on clinical outcomes of continuous ambulatory peritoneal dialysis patients in Taiwan -10 years' experience in a single center. Perit Dial Int 2014;34:85-94.

39 Szeto C-C, Kwan BC-H, Chow K-M, et al. Predictors of residual renal function decline in patients undergoing continuous ambulatory peritoneal dialysis. Perit Dial Int 2015;35:180-8.

40 Vilar E, Farrington K. Emerging importance of residual renal function in end-stage renal failure. Semin Dial 2011;24:487-94.

41 Raimann JG, Kitzler TM, Levin NW. Factors affecting loss of residual renal function(s) in dialysis. Contrib Nephrol 2012;178:150-6.

42 Harris SAC, Lamping DL, Brown EA, et al. Clinical outcomes and quality of life in elderly patients on peritoneal dialysis versus hemodialysis. Perit Dial Int 2002;22:463-70.

43 van Diepen ATN, van Esch S, Struijk DG, et al. The first peritonitis episode alters the natural course of peritoneal membrane characteristics in peritoneal dialysis patients. Perit Dial Int 2015;35:324-32. 